\title{
Centros integradores: UNA EXPERIENCIA DE ORDENAMIENTO TERRITORIAL EN EL ESTADO DE TABASCO
}

\author{
Baldemar Hernández Márquez, Judith Pérez Castro \\ y Efraín Pérez Cruz ${ }^{1}$
}

Fecha de recepción: 09 de febrero de 2015. Fecha de aceptación: 14 de agosto de 2015.

\begin{abstract}
RESUMEN
En este artículo los autores plantean la necesidad de hacer investigación a nivel microrregional con tendencia a la integración territorial, por la biodiversidad que se presentan entre cada una de las microrregiones de México, no tan sólo desde el punto de vista de sus recursos naturales, sino también de sus recursos humanos, lo que ha constituido desde siempre un gran obstáculo para la integración regional, a pesar de los múltiples esfuerzos que se han hecho con diferentes programas que han fracasado por muchas razones, pero una de las principales ha sido su falta de continuidad.
\end{abstract}

Palabras clave: centros integradores, ordenamiento territorial, integración regional, espacios geográficos, microrregiones.

Clasificación JEL: O18, P25, R11, R59.

\section{Integration Centers: A Territorial Planning EXPERIEnce in the State of Tabasco}

\begin{abstract}
In this article, the authors assert the need to research territorial integration at the microregional level, due to the fact that the biodiversity is so varied between each of the micro-regions in Mexico, not only in terms of natural resources, but also in terms of human resources. This has always constituted a major obstacle for regional integration, in spite of the multiple efforts made through numerous programs, which have failed for a variety of reasons, a principal one of which has been their lack of continuity.
\end{abstract}

Key Words: Integration centers, territorial planning, regional integration, geographic spaces, micro-regions.

Universidad Juárez Autónoma de Tabasco, México; Instituto de Investigaciones sobre la Universidad y la Educación de la unam, México; Universidad Juárez Autónoma de Tabasco, México, respectivamente. Correos electrónicos: baldemarh21@hotmail.com, pkjudith33@yahoo.com.mx y efrain_perezcruz@hotmail.com 


\section{CENTRES INTÉGRATEURS: UNE EXPÉRIENCE DE RÉGULATION TERRITORIALE DANS L'ÉTAT DE TABASCO \\ Résumé}

Dans cet article, les auteurs posent la nécessité de mener des recherches au niveau micro-régional qui tendent vers l'intégration territoriale, en raison de la biodiversité que présentent chacune des microrégions du Mexique, non seulement du point de vue de leurs ressources naturelles mais aussi de ses ressources humaines, laquelle a toujours constitué un obstacle majeur à l'intégration régionale, malgré les multiples efforts qui ont été faits à travers divers programmes, qui ont échoué pour de nombreuses raisons mais dont une des principales a été leur manque de continuité.

Mots clés: centres intégrateurs, régulation territoriale, intégration régionale, espaces géographiques, microrégions.

\section{CENTROS INTEGRADORES: UMA EXPERIÊNCIA DE ORDENAMENTO TERRITORIAL NO ESTADO DE TABASCO \\ Resumo}

Neste artigo os autores apresentam a necessidade de fazer uma pesquisa a nível micro regional com tendência à integração territorial, pela biodiversidade que presente entre cada uma das microrregiốes do México, não só do ponto de vista dos seus recursos naturais, mas também dos seus recursos humanos. Isso sempre foi um grande obstáculo para a integração regional, apesar dos múltiplos esforços que foram feitos com diferentes programas, que fracassaram por muitas razóes, mas que se explica principalmente pela falta de continuidade destes.

Palavras-chave: centros integradores, ordenamento territorial, integração regional, espaços geográficos, microrregiōes.

\section{融合中心：塔巴斯科州土地规划经验}

摘要

鉴于墨西哥小型区域所具备的自然资源及人力资源的双重生物多样性, 本文作者提出了在小型区域进行调查研究以促进当地的土地融合这一必要 性。小型区域的上述生物多样性一直以来都是区域融合的巨大障碍。目前 已在小型区域实施了不同的项目计划, 做了各种各样的尝试和努力, 但是 由于多种原因, 这些努力都以失败告终, 其中失败的主要原因是缺乏持续 性。

关键词：融合中心，土地规划，区域融合，地理空间，小型区域 


\section{INTRODUCCIÓN}

México se ha caracterizado por ser un país de las grandes desigualdades. Ya en el año de 1811, Alejandro Von Humboldt escribía en su Ensayo político sobre el reino de la Nueva España: "México es el país de la desigualdad. Acaso en ninguna parte la hay más espantosa en la distribución de fortunas, civilización, cultivo de la tierra y población [...]. Esta inmensa desigualdad de fortunas no sólo se observa en la casa de los blancos (europeos y criollos), sino que igualmente se manifiesta en los indígenas" (citado por Narro, Moctezuma y De la Fuente, 2013: 11). Esta situación se ha manifestado de manera recurrente en nuestro país y cada día son más grandes las desigualdades sociales y regionales.

No cabe la menor duda que se han hecho grandes esfuerzos por combatir esas grandes desigualdades y abatir la pobreza con programas sociales, como los que se realizaron en la etapa de crecimiento sostenido en el periodo de 1934-1970, sin embargo, no disminuyó el número de pobres. Entre 1934 y 1940 el Producto Bruto Interno (РІв) per cápita creció a una tasa media anual de $2.7 \%$. La población ocupada creció a una tasa media anual de $1.26 \%$, mientras que la Población Económicamente Activa (PEA) creció a una tasa media anual del $1.3 \%$; los precios crecieron a una tasa media de $6.3 \%$ anual y los salarios 3.6\% (Ayala, 1988: 214-245). Es decir la PEA y el empleo crecieron a tasas similares, pero los salarios reales bajaron un $2.7 \%$, ocasionando que bajara el poder adquisitivo de las familias hecho que impactó a los de más bajos ingresos, pero aunque no existen datos sobre la distribución del ingreso, es probable que el nivel de pobreza se haya modificado muy poco (Soria, 2000: 146).

En los años de 1941-1955, se inicia la etapa de industrialización del país que es financiada con los excedentes de la agricultura comercial a través del llamado "neolatifundismo" y se inicia el llamado despegue económico mediante una importante baja de los salarios, en tanto, la inflación se elevó a una tasa media anual de $11.8 \%$. El salario mínimo general bajó significativamente de 63.5 pesos de 1939 (con base 100 en 1978) a 31.1 pesos en 1955, o sea representó una caída de 51\%, mientras que la productividad en el sector industrial creció cerca de 50\% entre 1939 y 1952 (Bortz y Sánchez, 1985: 50). El salario real de 1939 no se recupera sino hasta 1965. La población ocupada creció entre 1940 y 1955 a un ritmo de 3.3\%, con lo que la economía fue capaz de crear nuevos empleos para la población creciente, pero el impacto del empobrecimiento se originó por la caída de los salarios.

En el periodo de 1956-1970 se instrumenta una política económica de crecimiento con estabilidad de precios, sin embargo, se ahonda la polarización 
entre la agricultura comercial y la campesina. El Estado mexicano cede en parte su papel de rectoría de dinamizador del crecimiento económico a la inversión extranjera, que inicia sus pasos para apropiarse de los sectores más dinámicos de la economía y promueve, indudablemente, la concentración industrial (Soria, 2000).

En la etapa de 1970-1987 se profundizan los desequilibrios entre las áreas rurales y urbanas, ante los problemas estructurales del sector industrial de responder a la pobreza global y en general por el agotamiento del patrón de acumulación por sustitución de importaciones, así como la indiferencia del sector industrial de la propuesta del gobierno de reforma fiscal, educativa y de la atención a la salud, que dan evidencias del inicio de la crisis estructural del sistema económico mexicano que demuestra su ineficacia a los cambios que se tenían que hacer necesariamente para insertarse en el mapa geográfico que estaba implicando la globalización, con la formación de bloques económicos, que obedecen a sus propios intereses, ante la incapacidad de capitalismo depredador que ha llegado a sus límites del crecimiento (Piketty, 2014).

\section{ALGUNAS CONSIDERACIONES TEÓRICAS}

El papel de la planificación tiene el propósito de enfrentarse a problemas que se presentan con distinta intensidad en diversas partes del espacio geográfico como son los que ocasiona el proceso de concentración espacial que advierte la necesidad de disponer de un marco teórico que sirva de fundamento para incorporar la dimensión espacial al plan (Kuklinski,1985).

Esta concentración origina grandes desigualdades que se observa en el proceso de globalización de la economía que es fuertemente asimétrica. A diferencia de lo que propugna el viejo paradigma de Centro-Periferia, es policéntrica y además las categorías Norte y Sur, en donde se ha perdido capacidad analítica, ya que los centros y las periferias en el nuevo orden internacional no se sitúan simétricamente a ambos lados de la hipotética línea divisora entre el Norte y Sur (Vásquez Barquero, 1990).

La teoría del desarrollo, pilar del libre intercambio y de la economía planificada, descansó en la explotación sin límites de los recursos del planeta, hasta que se descubre que esos recursos no son infinitos. Lo anterior marcó un cambio en la forma en que se venían organizando las regiones, con lo que se buscó que el desarrollo no se vinculara únicamente al crecimiento basado en la explotación de los recursos naturales y humanos, sino que este desarrollo se tradujera verdaderamente en el mejoramiento de los niveles de vida de la 
población y, lo más importante, coadyuvara en la conservación del medio ambiente (González, 1979).

Sin embargo, en la economía el significado del espacio no es un asunto trivial, se han identificado, cuando menos, cinco perspectivas distintas sobre este concepto (Isaac y Quintana, 2012). La primera proviene de la tradición económica europea, en la que se incorporó una concepción de espacio que lo definía como una barrera física para la actividad económica. La segunda emana de la teoría económica del crecimiento de los años cincuenta y sesenta, en la que se retomó la idea de espacio como un mero contenedor de la actividad económica.

En los años setenta, las interpretaciones sobre el espacio dan un giro importante. En la teoría del desarrollo, el espacio resultaba un factor de producción y, por consiguiente, una fuente de ventajas para las empresas. El espacio comenzó a ser visto como diversificado-relacional, en la medida en que el desarrollo se producía de forma heterogénea en áreas selectivas (Capello, 2007).

De este modo, el espacio-territorio representa el soporte de cualquier tipo de actividad humana por lo que su ordenamiento, así como el espacio cognitivo (Capello, 2006), no sólo se concreta a la ubicación y distribución de actividades productivas o núcleos de producción, sino que más bien apunta a una dimensión multifuncional, concretada en los acuerdos jurídicos que se originan del consenso político entre distintos agentes y niveles territoriales. La falta de planificación territorial se traduce en anarquía espacial, mal aprovechamiento de los recursos y el desconocimiento de sus potencialidades.

Concretamente, sobre la situación de América Latina, Montes (2001) apunta que cada vez más el tema del ordenamiento territorial se ha vuelto un issue central para las políticas nacionales las cuales se han abocado a lograr el equilibrio entre las variables económicas y sociales y las demandas sociales, la productividad y la conservación del medio ambiente. El ordenamiento territorial adquiere, según este autor, dos connotaciones conceptuales y metodológicas: una que, por el lado de la demanda, se orienta al estudio de los problemas socioeconómicos y políticos de la población establecida en un determinado territorio y otra que, por el lado de la oferta, se concentra en el análisis de las circunstancias y rasgos particulares del medio en el que se realizan las actividades de los actores sociales, así como en las probabilidades que ofrece para la satisfacción de la demanda.

En suma, el ordenamiento territorial es un conjunto de acciones concertadas para orientar la transformación, ocupación y utilización de los espacios geográficos, a partir de las necesidades e intereses de la población, las poten- 
cialidades del territorio y la armonía con el medio ambiente, todo esto con el fin de promover el desarrollo social y económico.

Cabe destacar que aunque el tema del ordenamiento territorial ha cobrado importancia en las últimas décadas, especialmente a la luz del crecimiento demográfico, la pauperización de los niveles de vida de gran parte de la población mundial y la problemática del medio ambiente, éste no es un objeto de estudio emergente en el campo de las ciencias sociales, al contrario, desde la primera mitad xx encontramos trabajos muy serios y relevantes, como el del geógrafo Walter Christaller que en los años treinta construye su teoría de los lugares centrales en Alemania y en el que se articulan los conceptos de lugar central, bien o servicio central y el área de influencia.

La centralidad de un lugar no depende del espacio sino de la función que tiene; es decir, existen una variedad de lugares centrales que van desde aquéllos que se especializan en funciones (por ejemplo, comercio, industria, entre otras), ubicuas o dispersas -que pueden encontrarse en varios lugares- $y$ en aquellos bienes $y$ servicios que son producidos centralmente.

Los conceptos de distancia económica y umbral también son importantes para entender la centralidad del lugar. La distancia económica es el trayecto que el consumidor está dispuesto a viajar para comprar el bien [...]. El umbral es la cantidad mínima de consumidores o demanda requerida para que alguna actividad económica emerja y se sostenga (Peńa, 2003: 188).

En ese mismo tenor, se encuentra el concepto de región económica de Lösch (1957), quien lo elabora como alternativa a los límites geográficos, políticos y culturales en los que tradicionalmente se dividen los estados y a los que consideraba artificiales y con pocas posibilidades de desarrollo.

La región económica toma como base la distribución de las actividades productivas dentro de un territorio, así como la dinámica que adquieren los procesos económicos dependiendo la distancia. En términos ideales, esta noción nos remite a un espacio continuo y homogéneo, económicamente independiente, con una distribución poblacional y de recursos uniforme que, por sus características, permite maximizar las utilidades y los beneficios de consumidores y productores. Las diferentes unidades productivas hexagonalmente se mantendrán estables para toda la población residente dentro de una misma área, pero tendrán que ir aumentando en la medida que se tengan que vender en zonas más cada vez más alejadas. El resultado final es la concentración económica de las actividades. 
Otros modelos de organización territorial importantes han sido los polos de desarrollo de Perroux (1955), que un lustro después sería concretado en el ámbito geográfico por Boudeville. Aquí, el énfasis se sitúa en encontrar un modelo de funcionamiento regional (Coraggio, 1972) de la demanda de producción localizada exógenamente, por el azar, por alguna autoridad pública o por alguna otra causa. También, está el modelo gravitacional que se orienta al análisis de los lugares centrales, sus jerarquías y las relaciones que entablan entre sí para entender la estructura general de las ciudades. Se le denomina gravitacional porque para esta perspectiva, al igual que en la ciencia física, existen espacios que ejercen un campo de gravedad sobre los individuos, los cuales dependen principalmente de la densidad o masa de la ciudad y la distancia, pero además de los flujos monetarios, los empleos, la migración, los productos y servicios que la caracterizan (Cárdenas y García, 2005). Asimismo, está el modelo de desarrollo regional integral que se apoya en la teoría del desarrollo endógeno-sustentable y sitúa a la planeación integradora de políticas y a la participación local como los principales medios tanto para la resolución de las demandas sociales como para la consecución del bienestar colectivo. Esta es una propuesta que, en gran medida, surge como una respuesta alternativa a los modelos de sustitución de importaciones y liberalizadores, seguidos principalmente por los países en vías de desarrollo, y que derivaron en el empobrecimiento de gran parte de la población, el endeudamiento nacional, el deterioro de los niveles de vida y el daño al medio ambiente.

Finalmente, cabría decir que en los análisis sobre organización territorial es necesario considerar el peso que tienen los factores social y geográfico-territorial pues, como señala Andrés (2013: 37):

[...]con el paso de la historia se producen cambios en la estructura social, se buscan nuevas funciones capaces de satisfacer las necesidades inherentes a cada momento, surgen nuevos procesos productivos, se teje una estructura territorial basada en modernos sistemas de conexión, la continua modernización de las estructuras sociales llevan a la conclusión de que los condicionantes básicos del primitivo emplazamiento acaban por convertirse en su principal disfuncionalidad.

Es decir, en la organización territorial, como en la mayoría de los procesos sociales, no existen modelos únicos y totalmente generalizables, sino que se deben tomar en cuenta los rasgos y necesidades de la población, las condiciones geográficas, las capacidades económicas, la vocación productiva, las vías de comunicación, la densidad de las relaciones sociales al interior y exterior de la comunidad, así como los cambios que con el tiempo se pueden producir en las demandas y en la organización comunitaria. 


\section{LA ORGANIZACIÓN TERRITORIAL Y EL DESARROLLO COMUNITARIO DE LOS CENTROS INTEGRADORES}

La idea de los centros integradores tiene su origen en los pueblos-hospitales implementados por don Vasco de Quiroga en Santa Fe, y posteriormente, en Michoacán a mediados del siglo xvi. Con ellos se pretendían reestructurar la vida comunitaria para abatir las grandes desigualdades sociales a partir de la integración de las familias, la incorporación de la mujer al trabajo con igualdad de condiciones que los hombres, la reorganización de los bienes, el trabajo colaborativo y la participación de gente de la ciudad en las actividades del campo (González, 1979). La palabra hospital aplicada a estos pueblos instituidos por Quiroga hacía referencia a la "hospitalidad", al servicio y al buen acogimiento brindado a todos aquellos que lo necesitaban.

Vasco de Quiroga utilizó la idea aristotélica del origen de las ciudades -aunque no la tomara de fuente directa- para dar cimiento a sus repúblicas indígenas " [...] La comunidad perfecta de varias aldeas ha dicho Aristóteles es la polis [...]. Una vez que el hombre ha formado familia y que varias familias se han unido para formar aldeas y cuando las aldeas se han juntado para formar la comunidad perfecta se llega a la polis" (González, 2006: 142143). Particularmente, en su trabajo en el estado de Michoacán, don Vasco de Quiroga buscó aprovechar el potencial de los recursos naturales de la zona para que, junto con la creatividad y los conocimientos acumulados que poseían las etnias, las personas desarrollaran sus capacidades y propiciaran el mejoramiento de la comunidad.

Los pueblos-hospitales constituyeron el primer ejemplo de desarrollo territorial que se tiene registrado en México, a pesar de que ese término no se hubiese acuñado aún en los tiempos de la Colonia. Fue hasta después de muchos años cuando se vuelven a registrar otras experiencias de desarrollo regional que, aunque tenían fines diferentes, partían del concepto de ordenamiento territorial como resultado de la evolución conceptual de la planificación (Bettelheim, 1965), que en México tuvo sus antecedentes con el desarrollo de las cuencas hidrológica, 1946-1952 (Carrillo, 2010), y que más adelante se conocería con el nombre de planificación regional y territorial (Cortez y Delgadillo, 2008).

A mediados de los años ochenta, en el estado de Tabasco, se comienza a diseñar un modelo de desarrollo endógeno fundamentado en la integración territorial de las regiones. Este proyecto se enfocaba en la identidad cultural y en la vocación productiva de los recursos naturales humanos, que fungían como eje fundamental para el crecimiento y el desarrollo de la microrregión. 
En ese sentido, los centros integradores, como se les conocería posteriormente, nacen como una respuesta gubernamental para configurar una estructura organizacional que permita la racionalización de los recursos públicos y mejorar la atención a una población bastante dispersa y con necesidades muy específicas, en donde el papel del Estado, a través de políticas públicas es como responde a las necesidades de la población en una sociedad bien organizada (Bustamante, 2008).

En el presente artículo exponemos parte de los resultados de una investigación cuyo fin fue realizar un análisis comparativo de estos centros integradores para observar cuáles han sido los principales cambios que se han generado a casi tres décadas de que se inició este proyecto de organización territorial y proponer un modelo para potenciar el desarrollo de estas microrregiones.

\section{LA CONFORMACIÓN DE LOS CENTROS INTEGRADORES EN TABASCO}

El estado de Tabasco se encuentra ubicado en el sureste de la República mexicana, en donde se llevó a cabo el modelo de centros integradores en el año de 1985, a partir de una iniciativa de descentralización de los servicios administrativos a nivel de los gobiernos municipal y estatal (Gobierno del Estado de Tabasco, 1988).

Figura 1. Ubicación geogrática del estado de Tabasco del sureste mexicano

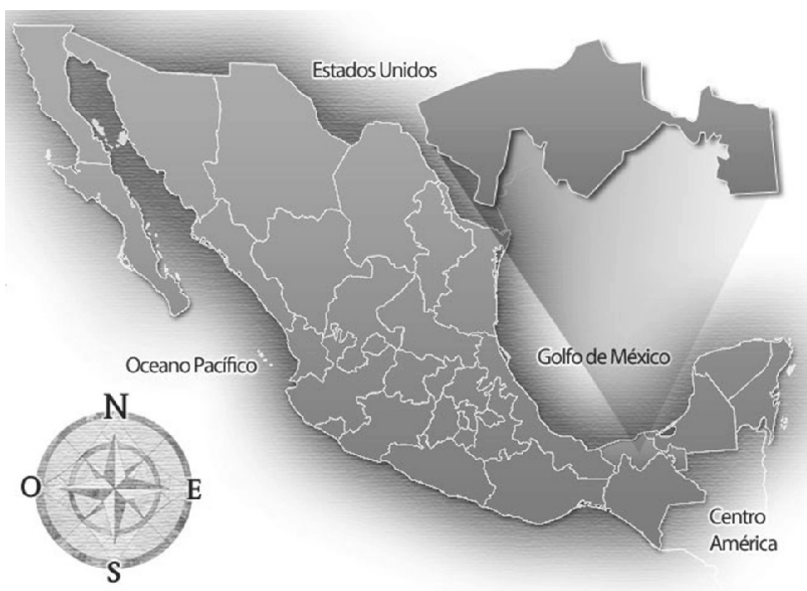

Fuente: elaboración propia para una presentación del Proyecto Centros Integradores. Conacyt - FOMIX Gobierno del Estado de Tabasco. 
Cuando comenzaron a constituirse, el estado tenía alrededor de 1400 comunidades dispersas por todo el territorio (INEGI, 1980), lo que dificultaba tanto la implementación de las políticas públicas como la provisión de productos y servicios.

Se decidió orientar las acciones gubernamentales hacia algunos poblados que, por diversas razones, se habían convertido en polos de atracción para otras comunidades; éstos actuarían como el corazón de los centros integradores, a ellos llegaría el abasto de mercancías, los servicios de atención médica de primer y segundo nivel, las instituciones de educación básica y media superior y los demás servicios comunitarios. El objetivo era que la población rural no tuviera que desplazarse hasta las cabeceras municipales o, incluso, hasta la capital del estado para cubrir sus necesidades más elementales (Gobierno del Estado de Tabasco, Manual de Organización de los Centros Integradores, 1984: 12).

En este proceso se tomó en cuenta la ubicación de las comunidades, sus características geográficas, sus recursos naturales y su vocación productiva en lo que respecta a los suelos (Palma y Sánchez, 2002). Otros factores fueron los usos, costumbres y conocimientos tradicionales compartidos. Así, los centros integradores, como polos de desarrollo microrregional, poco a poco permitirían ir ampliando la cobertura de los servicios públicos y hacer eficiente el uso de los recursos. Para ello, el gobierno estableció dos estrategias principales: 1) introducir cambios en la estructura territorial y poblacional para alcanzar la integración, y 2) rescatar las potencialidades de las diferentes regiones del estado estado (Gobierno del Estado de Tabasco, 1983b).

Para la primera, se emprendió la construcción y el mantenimiento de la infraestructura, en especial, se hizo énfasis en la edificación de puentes y carreteras para comunicar a todas aquellas poblaciones que por mucho tiempo habían permanecido aisladas. Posteriormente, esta estrategia se orientó hacia la parte social, lo que implicó la colaboración entre los miembros de las diferentes comunidades y los sectores gubernamentales para definir las prioridades de cada microrregión.

Para la segunda estrategia, las acciones se orientaron a aprovechar las ventajas productivas, a fin de disminuir la dependencia del petróleo y diversificar las actividades económicas. Para cada centro integrador se identificó un conjunto de recursos y/o actividades económicas de acuerdo con su contexto, además de capacidades, usos y costumbres (Gobierno del Estado de Tabasco, 1983a).

En suma, se trataba de unir lo disperso partiendo de un ordenamiento territorial como disciplina y como instrumento de políticas públicas, con un sentido de estrategia y de defensa de los recursos físicos existentes, así como de la apropiación de las fuentes naturales de riqueza (Cortez y Delgadillo, 2008: 56). 
Figura 2. Ubicación geográfica de los centros integradores del estado de Tabasco

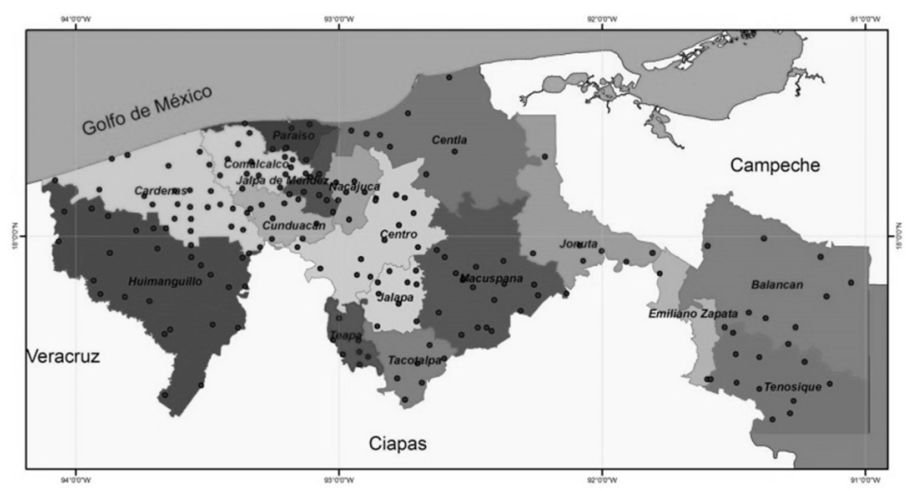

Leyenda

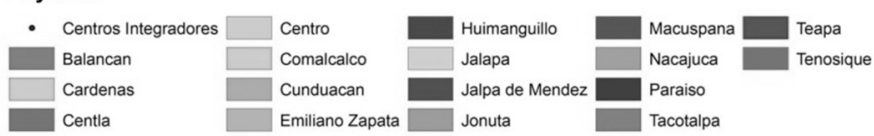

Fuente: elaboración con datos del Proyecto Centros Integradores. Conacyt - FOMIX Gobierno del Estado de Tabasco.

El método de trabajo de los centros integradores se sustentó jurídicamente en la Constitución Política de los Estados Unidos Mexicanos que, en su Artículo 26, sitúa a la planeación democrática como base para el desarrollo nacional, para la atención de las demandas sociales y para promover la participación de los diversos sectores poblacionales. El otro pilar fue la Constitución Política del Estado Libre y Soberano de Tabasco, vigente en la década de los ochenta, que en su Artículo 76 apuntaba que la entidad tenía la capacidad de diseñar un sistema de planeación democrática que otorgara dinamismo, solidez, permanencia y equidad a los diferentes ámbitos de la vida estatal, económico, político, social y cultural. Asimismo, se destacaba el papel que los municipios jugaban en la planeación democrática a través de la elaboración de sus planes trianuales y sus programas operativos anuales. Es decir, que en este proceso se pretendía que los diferentes órdenes de gobierno y la propia gente de la comunidad tomaran parte en la definición de los objetivos, metas y prioridades de los centros integradores.

Con esta base jurídica, en 1985 se inició la distribución territorial de las microrregiones. El punto de partida fue la cartografía de cada municipio y la ubicación de cada localidad, considerando además su identidad cultural, su cercanía geográfica y la relación social y comercial con el centro integrador. 
Finalmente, los centros integradores quedaron establecidos de la siguiente manera: el municipio de Balancán con 9 centros integradores, Cárdenas con 25, Centla con 8, Centro con 13, Comalcalco con 13, Cunduacán con 10, Emiliano Zapata con 2, Huimanguillo con 26, Jalapa con 9, Jalpa de Méndez con 8, Jonuta con 7, Macuspana con 19, Nacajuca con 6, Paraíso con 7, Tacotalpa con 6, Teapa con 7 y Tenosique con 11 (Gobierno del Estado de Tabasco, 1988). En total, 185 centros integradores distribuidos en los 17 municipios del estado.

\section{LA INVESTIGACIÓN SOBRE LOS CENTROS INTEGRADORES}

En el proceso de la investigación desarrollamos un modelo de información que permitiera hacer un inventario de las potencialidades y la vocación productiva, así como de los recursos naturales y humanos de las distintas microrregiones. El trabajo de gabinete y de campo buscó recuperar información sobre la dinámica sociodemográfica, la economía y los servicios públicos de las comunidades para después hacer las comparaciones entre los diferentes centros integradores. Igualmente, diseñamos dos instrumentos que se aplicaron en las cuatro regiones en que tradicionalmente se ha dividido el estado de Tabasco: Centro, Chontalpa, Sierra y Ríos y que comprenden a los 185 centros integradores. El primero estaba compuesto por las siguientes secciones: 1) rasgos generales del centro integrador, 2) localización en sus coordenadas geográficas, 3) entorno físico, 4) administración, 5) localidades que conformaban el centro integrador, 6) composición poblacional, 7) infraestructura de servicios públicos, 8) infraestructura productiva, 9) áreas de esparcimiento, 10) climatología y 11) vocación productiva. El segundo instrumento se utilizó específicamente para conocer las condiciones de los servicios educativos y estaba integrado por los siguientes apartados: 1) rasgos generales del centro integrador, 2) autoridades educativas, 3) niveles educativos con que cuenta el centro integrador, 4) infraestructura educativa, 5) población estudiantil, 6) personal docente, administrativo y de intendencia, 7) capacidad de atención a la demanda y 8) necesidades educativas especiales.

Con la información, se construyeron dos bases de datos, utilizando el programa estadístico spss, la primera, sobre las condiciones socioeconómicas, con 142 variables y 186 casos, y la segunda, sobre la situación educativa, con 107 variables y 717 casos. Los informantes clave fueron los delegados municipales, las autoridades educativas, los presidentes de la sociedad de padres de familia, los representantes de los comités de acción comunitaria, algunos miembros de 
organizaciones no gubernamentales y otras personas reconocidas como líderes de la comunidad.

Por último, se organizaron tres talleres para el manejo de la información y un diplomado en spss a partir de los cuales se obtuvieron los resultados finales de la investigación.

\section{CONTRASTE ENTRE LOS CENTROS INTEGRADORES AYER Y HOY}

Cuando emprendimos esta investigación nos preguntábamos cuáles eran los cambios más importantes que se habían operado en los centros integradores desde sus inicios, a mediados de los años ochenta hasta la fecha. Si el paso del tiempo había detenido su desarrollo o, si por el contrario, los centros integradores se habían adaptado y transformado para responder a las nuevas demandas de la población. Finalmente, queríamos saber si era posible construir un modelo de integración territorial, basado en esta experiencia, que permitiera aprovechar la vocación productiva y los recursos naturales y humanos. Inicialmente, nos planteamos, a manera de hipótesis, que se habían generado desigualdades significativas entre las microrregiones, en gran medida, como resultado de las diferencias que aún se observan entre las distintas regiones del país y también por la inconsistencia de las políticas públicas, lo que a su vez había provocado el agotamiento de este modelo de organización y de desarrollo regional. Es decir, al igual que Andrés (2013), asumíamos que el tiempo va creando nuevas necesidades y reestructurando las estructuras sociales, de tal forma que la organización se va desgastando hasta hacerse cada vez menos funcional para la propia comunidad, lo que a la postre deriva en el abandono del modelo o en la construcción de uno nuevo más congruente con las demandas emergentes.

Es importante señalar que para nuestro modelo de análisis, se hicieron las pruebas ANOva ${ }^{2}$ y la DUNCAN. Primero, para encontrar si había diferencias significativas entre los centros integradores a nivel municipal (ANOvA) y luego para indagar a qué nivel de significancia estaban esas diferencias (DUNCAN) y poder agrupar a los centros integradores según sus características. De esta manera, con respecto al promedio general de población, la prueba DUNCAN nos permitió identificar tres subconjuntos. El primero comprendió los municipios

2 Las pruebas ANOVA y DUNCAN son estadísticos que utilizamos para medir las diferencias significativas entre las medias poblacionales de los centros integradores. 
que contaban con menor población por centro integrador, como Tenosique con 601 habitantes, Jonuta con 913 y Jalapa con 986. En el segundo grupo, se localizaron los centros integradores de los municipios de Huimanguillo, Teapa, Tacotalpa, Balancán, Paraíso, Cunduacán y Macuspana, que en promedio tienen entre 1500 y 2500 habitantes. El último subconjunto abarcó a los municipios cuyos centros integradores tienen la mayor densidad poblacional, como Cárdenas, Centla, Jalpa de Méndez, Nacajuca, Comalcalco, Emiliano Zapata y Centro que en promedio poseen entre 2500 y 5000 habitantes (véase cuadro 1).

Con lo que respecta al grado de marginación, después de realizar otra prueba DUNCAN, encontramos también tres subconjuntos, el primero, con los municipios que tienen centros integradores con bajo índice marginación promedio, conformado por Jalapa, Paraíso y Centro. Con marginación media, están los municipios del segundo grupo integrado por Emiliano Zapata, Jalpa de Méndez, Nacajuca, Comalcalco, Cunduacán, Balancán y Macuspana. Finalmente, se encuentran los municipios que tienen centros integradores con un índice de marginación alto, como Jonuta, Cárdenas, Huimanguillo, Tenosique, Tacotalpa, Centla y Teapa. Fundamentalmente, este último se destaca por ser el único en el que todos sus centros integradores tienen un índice de marginación alto (véanse cuadro 2 y figura 3 ).

Para indagar sobre la infraestructura educativa, se levantó información sobre una muestra de 717 escuelas de los distintos centros integradores. Los municipios que contaban con el mayor número de escuelas en sus centros integradores fueron Huimanguillo con 96 instituciones, Cárdenas con 92 y Macuspana con 72 . En conjunto eran el 36.2\% del total las escuelas distribuidas en todos los centros integradores (véase cuadro 3 ).

De las 717 escuelas, las de nivel primaria representan la mayor proporción con $34 \%$, le siguen de cerca las de nivel preescolar con $31.1 \%$, después las de nivel secundaria con $24.5 \%$ y finalmente las de nivel medio superior con $10.3 \%$ (véase cuadro 4 ). 
Cuadro 1. Población de los centros integradores 2010

\begin{tabular}{|c|c|c|c|c|}
\hline \multirow[t]{2}{*}{ Municipio } & \multirow[t]{2}{*}{ C.I. } & \multicolumn{3}{|c|}{ Subconjunto } \\
\hline & & 1 & 2 & 3 \\
\hline Tenosique & 11 & 601.55 & & \\
\hline Jonuta & 7 & 913.57 & & \\
\hline Jalapa & 9 & 986.11 & & \\
\hline Huimanguillo & 26 & & 1549.81 & \\
\hline Teapa & 7 & & 1551.43 & \\
\hline Tacotalpa & 6 & & 1675.00 & \\
\hline Balancán & 10 & & 1710.80 & \\
\hline Paraíso & 7 & & 2045.14 & \\
\hline Cunduacán & 9 & & 2528.22 & \\
\hline Macuspana & 19 & & 2545.42 & \\
\hline Cárdenas & 25 & & & 2762.72 \\
\hline Centla & 8 & & & 2891.63 \\
\hline Jalpa de Menéndez & 8 & & & 2952.88 \\
\hline Nacajuca & 6 & & & 3030.67 \\
\hline Comalcalco & 13 & & & 3166.77 \\
\hline Emiliano Zapata & 2 & & & 3356.00 \\
\hline Centro & 13 & & & 5548.15 \\
\hline
\end{tabular}

*Significancia al 0.004 .

Fuente: elaboración propia con información recaba del Proyecto Centros Integradores. Conacyt - FOMIX Gobierno del Estado de Tabasco. 
Baldemar Hernández Márquez, Judith Pérez Castro y Efraín Pérez Cruz

Cuadro 2. Grados de marginación de los centros integradores a nivel municipal

\begin{tabular}{|c|c|c|c|c|}
\hline \multirow[t]{2}{*}{ Municipio } & \multirow[t]{2}{*}{ C.I. } & \multicolumn{3}{|c|}{ Subconjunto } \\
\hline & & 1 & 2 & 3 \\
\hline Jalapa & 9 & 1.33 & & \\
\hline Paraíso & 7 & 1.57 & 1.57 & \\
\hline Centro & 13 & 1.69 & 1.69 & \\
\hline Emiliano Zapata & 2 & 2.00 & 2.00 & \\
\hline Jalpa de Méndez & 8 & 2.00 & 2.00 & \\
\hline Nacajuca & 6 & 2.00 & 2.00 & \\
\hline Comalcalco & 13 & & 2.08 & \\
\hline Cunduacán & 9 & & 2.33 & \\
\hline Balancán & 10 & & 2.40 & \\
\hline Macuspana & 19 & & 2.42 & \\
\hline Jonuta & 7 & & & 2.57 \\
\hline Cárdenas & 25 & & & 2.64 \\
\hline Huimanguillo & 26 & & & 2.77 \\
\hline Tenosique & 11 & & & 2.82 \\
\hline Tacotalpa & 6 & & & 2.83 \\
\hline Centla & 8 & & & 2.87 \\
\hline Teapa & 7 & & & 3.00 \\
\hline
\end{tabular}

*Significancia al 0.004 .

Fuente: elaboración propia con información recaba del Proyecto Centros Integradores. Conacył - FOMIX Gobierno del Estado de Tabasco. 
Centros integradores: una experiencia de ordenamiento territorial en el estado de Tabasco

Cuadro 3. Número de escuelas de los centros integradores a nivel municipal

\begin{tabular}{lcc}
\hline Municipios & Escuelas & Porcentaje \\
\hline Balancán & 37 & 5.2 \\
Cárdenas & 92 & 12.8 \\
Centla & 35 & 4.9 \\
Centro & 64 & 8.9 \\
Comalcalco & 59 & 8.2 \\
Cunduaćn & 35 & 4.9 \\
Emiliano Zapata & 9 & 1.3 \\
Huimanguillo & 96 & 13.4 \\
Jalapa & 29 & 4.0 \\
Jalpa de Méndez & 32 & 4.5 \\
Jonuta & 23 & 3.2 \\
Macuspana & 72 & 10.0 \\
Nacajuca & 24 & 3.3 \\
Paraíso & 27 & 3.8 \\
Tacotalpa & 25 & 3.5 \\
Teapa & 22 & 3.1 \\
Tenosique & 36 & 5.0 \\
Total & 717 & 100,0 \\
\hline
\end{tabular}

Fuente: elaboración propia con información recaba del Proyecto Centros Integradores. Conacyt - FOMIX Gobierno del Estado de Tabasco.

Cuadro 4. Nivel educativo de las escuelas por centro integrador a nivel municipal

\begin{tabular}{lcc}
\hline Nivel educativo & Escuelas & Porcentaje \\
\hline Preescolar & 223 & 31.1 \\
Primaria & 244 & 34.0 \\
Secundaria & 176 & 24.5 \\
Preparatoria & 74 & 10.3 \\
Total & 717 & 100.0 \\
\hline
\end{tabular}

Fuente: elaboración propia con información recaba del Proyecto Centros Integradores. Conacyt - FOMIX Gobierno del Estado de Tabasco. 


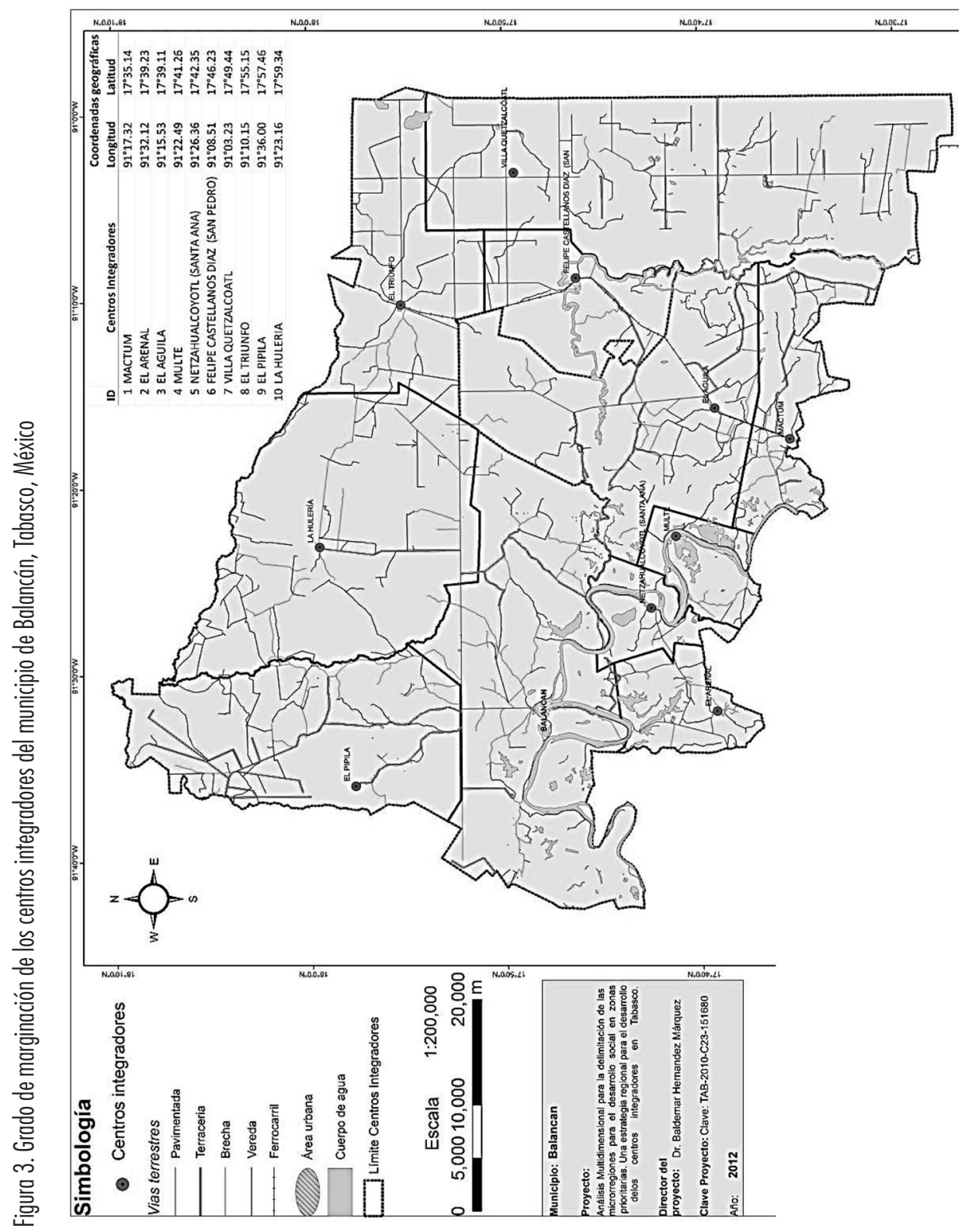




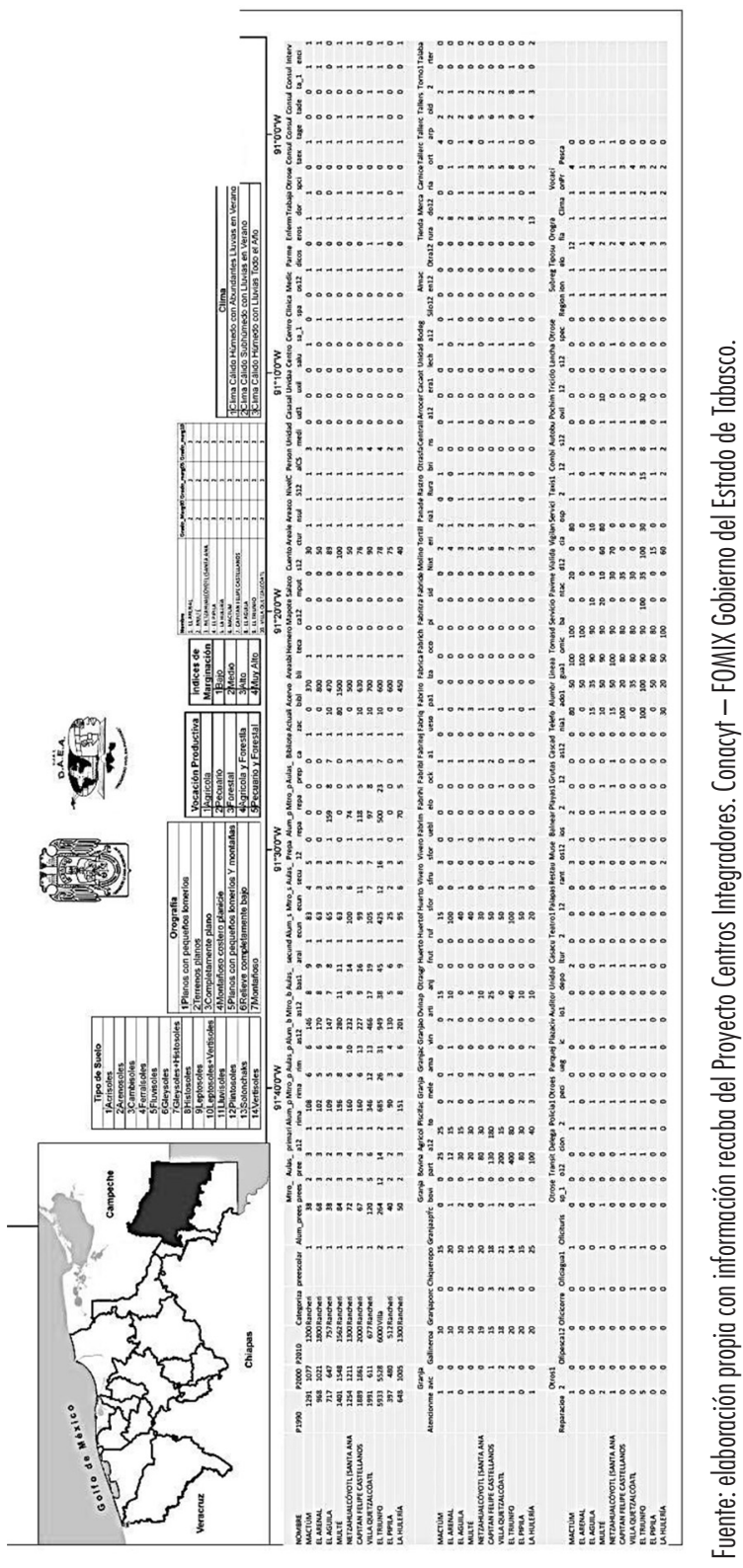


Para valorar las diferencias en cuanto a los servicios educativos por centro integrador realizamos la prueba DUNCAN, donde nuevamente se pudieron identificar tres subgrupos. El primero donde se ubicaron los centros integradores de los municipios de Macuspana, Centro, Balancán, Centla y Emiliano Zapata, en los que la mayoría de sus centros integradores tenían escuelas para todos los niveles educativos y contaban con más de una institución del nivel medio superior. En el segundo están aquellos que disponen instituciones para todos los niveles educativos y la mayoría de sus centros integradores tienen por lo menos una escuela del nivel medio superior, ya sea colegio de bachilleres o centro de bachillerato tecnológico agropecuario, como Jalapa, Jalpa de Méndez, Nacajuca, Cárdenas, Huimanguillo, Jonuta, Cunduacán y Comalcalco. Y, finalmente, el tercero que comprendió los municipios de Paraíso, Tacotalpa, Teapa y Tenosique cuya oferta educativa llegó nada más hasta la secundaria, es decir, ninguno de sus centros integradores poseía una escuela de educación media superior (véase cuadro 5).

En cuanto a las delegaciones municipales, que ejercen una importante función en cuanto a la representación de las comunidades, la gestión de los recursos, el cumplimiento de las leyes y disposiciones de los ayuntamientos, la supervisión de los servicios públicos y la organización comunitaria en general, en los centros integradores cuentan con oficinas para el delegado municipal, Registro Civil, Tesorería del municipio, oficinas para el pago de la luz eléctrica de la Comisión Federal de Electricidad, de agua potable y alcantarillado, Oficina de Pesca, supervisores escolares. La prueba DunCan nos arrojó dos subconjuntos. El primero donde se encuentran los municipios de Jalpa de Méndez, Jalapa, Tenosique, Nacajuca, Centro, Teapa, Cunduacán, Balancán, Comalcalco, Macuspana y Huimanguillo, en los que algunos de sus centros integradores no cuentan con delegación. Y el segundo que abarca los municipios de Cárdenas, Centla, Emiliano Zapata, Jonuta, Paraíso y Tacotalpa, en los que la totalidad de sus centros integradores tienen sus propias delegaciones (véase cuadro 6).

Finalmente, quisimos indagar sobre la vocación productiva de los centros integradores. Aquí, la prueba DUNCAN nos permitió identificar cuatro subconjuntos. En el primero, donde la mayoría de los centros integradores tienen una vocación agrícola, están los municipios de Emiliano Zapata y Tenosique. En el segundo están los municipios de Cunduacán, Balancán, Huimanguillo, Jalapa y Nacajuca, cuyos centros integradores en su mayoría tienen vocación pecuaria-agrícola y forestal. El tercer grupo abarca a los centros integradores con una vocación productiva agrícola y forestal y se conforma por los municipios de Tacotalpa, Jalpa de Méndez, Jonuta, Paraíso, Centro y Cárdenas. 
Cuadro 5. Promedio de escuelas preparatorias en los centros integradores a nivel municipal

\begin{tabular}{|c|c|c|c|c|}
\hline \multirow[t]{2}{*}{ Municipio } & \multirow[t]{2}{*}{ C.I. } & \multicolumn{3}{|c|}{ Subconjunto } \\
\hline & & 1 & 2 & 3 \\
\hline Paraíso & 7 & 0.00 & & \\
\hline Tacotalpa & 6 & 0.00 & & \\
\hline Teapa & 7 & 0.00 & & \\
\hline Tenosique & 11 & 0.00 & & \\
\hline Jalapa & 9 & .11 & 11 & \\
\hline Jalpa de Méndez & 8 & .25 & .25 & .25 \\
\hline Nacajuca & 6 & .33 & .33 & .33 \\
\hline Cárdenas & 25 & .40 & .40 & .40 \\
\hline Huimanguillo & 26 & .42 & .42 & .42 \\
\hline Jonuta & 7 & .43 & .43 & .43 \\
\hline Cunduacán & 9 & .44 & .44 & .44 \\
\hline Comalcalco & 13 & .46 & .46 & .46 \\
\hline Macuspana & 19 & .53 & .53 & .53 \\
\hline Centro & 13 & .54 & .54 & .54 \\
\hline Balancán & 10 & & .60 & .60 \\
\hline Centla & 8 & & & .75 \\
\hline Emiliano Zapata & 2 & & & 1.00 \\
\hline
\end{tabular}

Fuente: elaboración propia con información recaba del Proyecto Centros Integradores. Conacyt - FOMIX Gobierno del Estado de Tabasco.

Por último, están los municipios de Cárdenas, Centla, Comalcalco, Teapa y Macuspana que poseen la vocación productiva agrícola-forestal y pecuariaforestal (véase cuadro 7). 
Baldemar Hernández Márquez, Judith Pérez Castro y Efraín Pérez Cruz

Cuadro 6. Delegaciones de los centros integradores a nivel municipal

\begin{tabular}{|c|c|c|c|}
\hline \multirow[t]{2}{*}{ Municipio } & \multirow[t]{2}{*}{ C.I. } & \multicolumn{2}{|c|}{ Subconjunto } \\
\hline & & 1 & 2 \\
\hline Jalpa de Méndez & 8 & .63 & \\
\hline Jalapa & 9 & .67 & .67 \\
\hline Tenosique & 11 & .82 & .82 \\
\hline Nacajuca & 6 & .83 & .83 \\
\hline Centro & 13 & .85 & .85 \\
\hline Teapa & 7 & .86 & .86 \\
\hline Cunduacán & 9 & .89 & .89 \\
\hline Balancán & 10 & .90 & .90 \\
\hline Comalcalco & 13 & .92 & .92 \\
\hline Macuspana & 19 & .95 & .95 \\
\hline Huimanguillo & 26 & .96 & .96 \\
\hline Cárdenas & 25 & & 1.00 \\
\hline Centla & 8 & & 1.00 \\
\hline Emiliano Zapata & 2 & & 1.00 \\
\hline Jonuta & 7 & & 1.00 \\
\hline Paraíso & 7 & & 1.00 \\
\hline Tacotalpa & 6 & & 1.00 \\
\hline
\end{tabular}

*Significancia 0.004 .

Fuente: elaboración propia con información recaba del Proyecto Centros Integradores. Conacyt - FOMIX Gobierno del Estado de Tabasco. 
Cuadro 7. Vocación productiva de los centros integradores a nivel municipal

\begin{tabular}{|c|c|c|c|c|c|}
\hline \multicolumn{6}{|c|}{$\begin{array}{l}\text { Vocación productiva } \\
\text { DUNCAN }\end{array}$} \\
\hline \multirow[t]{2}{*}{ Municipio } & \multirow[t]{2}{*}{ N } & \multicolumn{4}{|c|}{ Subconjunto } \\
\hline & & 1 & 2 & 3 & 4 \\
\hline Emiliano Zapata & 2 & 1.50 & & & \\
\hline Tenosique & 11 & 1.91 & 1.91 & & \\
\hline Cunduacán & 9 & 2.33 & 2.33 & 2.33 & \\
\hline Balancán & 10 & 2.40 & 2.40 & 2.40 & 2.40 \\
\hline Huimanguillo & 26 & 2.50 & 2.50 & 2.50 & 2.50 \\
\hline Jalapa & 9 & 2.67 & 2.67 & 2.67 & 2.67 \\
\hline Nacajuca & 6 & 2.67 & 2.67 & 2.67 & 2.67 \\
\hline Tacotalpa & 6 & & 3.33 & 3.33 & 3.33 \\
\hline Jalpa de Méndez & 8 & & & 3.63 & 3.63 \\
\hline Jonuta & 7 & & & 3.71 & 3.71 \\
\hline Paraíso & 7 & & & 3.71 & 3.71 \\
\hline Centro & 13 & & & 3.92 & 3.92 \\
\hline Cárdenas & 25 & & & & 4.00 \\
\hline Centla & 8 & & & & 4.00 \\
\hline Comalcalco & 13 & & & & 4.23 \\
\hline Teapa & 7 & & & & 4.29 \\
\hline Macuspana & 19 & & & & 4.53 \\
\hline
\end{tabular}

**Significancia 0.004 .

Fuente: elaboración propia con información recaba del Proyecto Centros Integradores. Conacyt - FOMIX Gobierno del Estado de Tabasco. 


\section{CONSIDERACIONES FINALES}

Los centros integradores, como modelo de integración territorial, han sido pertinentes para muchas comunidades del estado de Tabasco a pesar de que, como hemos dicho, no hubo continuidad en las políticas gubernamentales. A la fecha, las microrregiones han seguido funcionando, con ciertas desigualdades importantes entre ellas que se reflejan en el crecimiento de su población, en los servicios públicos con los que cuentan, en los niveles de marginación y en sus condiciones educativas. A lo largo de esta investigación también encontramos algunos factores muy favorables que valdría la pena tomar en cuenta. Por ejemplo, algunos centros integradores han registrado un crecimiento significativo en su población, al grado que alcanzan cerca de los 5 mil habitantes. Otra cuestión ha sido el desarrollo de su capacidad de gestión lo que, tomando en cuenta el modelo de organización que aún conservan, les ha permitido mejorar y aumentar su infraestructura, obras y servicios públicos. Esto último, con el apoyo de políticas focalizadas permitiría a estos centros integradores generar un desarrollo sustentable y ser autosuficientes no sólo en materia alimentaria, sino competir en los mercados nacional e internacional.

Una estrategia para lograr el desarrollo podría ser el fortalecimiento del espacio cognitivo de las microrregiones a través de un modelo educativo que rescate su vocación productiva. Un proyecto de esta naturaleza podría iniciarse con los centros integradores que cuentan con mejor infraestructura educativa y que pueden aprovechar el potencial de los recursos humanos. Esto también podría evitar la migración por falta de empleo, que suele darse de manera muy marcada en aquellas microrregiones que no pueden absorber los recursos humanos capacitados.

Finalmente, es necesario estimular la inversión pública y privada, nacional y foránea, basada en estudios de factibilidad con la participación social de la población, que permita una distribución equitativa de la riqueza que se genere en su propio entorno de manera autosostenible, con tendencia a modular los desequilibrios entre las microrregiones de los centros integradores. En este sentido se considera que esta organización territorial constituye una opción viable para la integración de comunidades dispersas como una forma de aprovechar de manera eficiente las potencialidades primarias de sus recursos productivos, aprovechando la calidad de la mano de obra disponible en las propias comunidades, mejorando el nivel de ingresos de la población, que de otra manera están emigrando a la capital del estado, al centro de la República o como braceros a Estados Unidos, en todos estos casos sin ninguna posibilidad que garanticen que mejorarán sus condiciones de vida, como está suce- 
diendo en algunos centros integradores en donde han disminuido los niveles de marginación, a pesar de no haber continuidad en su trayectoria, que es lo que sugiere en este artículo.

\section{BIBLIOGRAFÍA}

Andrés, José (2013), "Equipamiento comercial y organización territorial”, Papeles de Geografía, (57-58), pp. 35-53.

Ayala, José (1988), Estado y desarrollo. La formación de la economía mixta mexicana, (1920-1982), México, FCE/SEMIP.

Bettelheim, Charles (1965), Problemas teóricos y prácticos de la planificación, Madrid, Tecnos.

Bortz, Jeffrey y Rafael Sánchez (1985), "Salarios y crisis económica en México", en J. Bortz et al., La estructura de salarios en México, México, UAM-sTPs.

Bustamante, Carlos (2008), Actores urbanos y politicas públicas. Estrategias de los manufactureros de la Ciudad de México ante el neoliberalismo, México, unAM-IIEc-Miguel Ángel Porrúa.

Capello, Roberta (2006), "La economía regional tras cincuenta años: desarrollos teóricos recientes y desafíos futuros", Investigaciones regionales (9), pp. 169-192.

(2007), Regional Economics, New York, Routledge.

Cárdenas, Mauricio y Camilo García (2005), "El modelo gravitacional y el TLC entre Colombia y Estados Unidos", Colombia Coyuntura Económica, 35 (1), pp. 47-72.

Carrillo, Ricardo (2010), Experiencias y perspectivas del desarrollo regional en México. Conferencia magistral presentada en el $15^{\circ}$ Encuentro Nacional sobre Regional en México: AMECIDER 2010, "Situación actual del pais: Politicas Públicas y Desarrollo Regional Sustentable”, Villahermosa, Tabasco. 18 de noviembre.

Coraggio, José Luis (1972), "Hacia una revisión de la teoría de los polos de desarrollo", Revista Latinoamericana de Estudios Urbano Regionales, 2(4), pp. 25-39.

Cortez, Héctor y Javier Delgadillo (2008), "Alcances del ordenamiento territorial en la planeación del desarrollo. Elementos conceptuales", en J. Delgadillo (coord.), Política territorial en México. Hacia un modelo de desarrollo basado en el territorio, pp. 55-105, México, Plaza y Valdés.

Gobierno del Estado de Tabasco (1983a), Plan Estatal de Desarrollo 19831988, Tabasco, México, Publicaciones del Estado de Tabasco. 
(1983b), Planes normativos de la región del Grijalva y del Usumacinta, Tomo I, México, Publicaciones del Estado de Tabasco.

(1984), Manual de Organización de los Centros Integradores, Tabasco, México, Publicaciones del Estado de Tabasco.

(1988), Memoria del Gobierno del Estado 1983-1988, Tabasco, México, Publicaciones del Estado de Tabasco.

González Pedrero, Enrique (1979), La riqueza de la pobreza. Apuntes para un modelo mexicano de desarrollo, México, Cuadernos de Joaquín Mortiz.

(2006), La cuerda floja, México, Fondo de Cultura Económica.

INEgi (1980), Censo General de Población y Vivienda 1980, México, INEgi. Consultado en http://www3.inegi.org.mx/sistemas/tabuladosbasicos/default.aspx?c=16762\&s=es septiembre de 2014 .

Isaac, Jorge y Luis Quintana (2012), "Industria y vaciamiento productivo regional en México”, en J. Calva (coord.), Desarrollo regional y urbano. Análisis estratégico para el desarrollo, vol. 13, pp. 39-34, México, Juan Pablos.

Kuklinski, Antoni (1985), Desarrollo polarizado y politicas regionales: en homenaje a Jacques Boudeville, México, Fondo de Cultura Económica.

Lösch, August (1957), Teoría económica espacial, Madrid, El Ateneo.

Montes, Pedro (2001), El ordenamiento territorial como opción de politicas urbanas y regionales en América Latina y el Caribe, Santiago de Chile, Cepal.

Narro, José, David Moctezuma y Diego de la Fuente (2013), "Descalabros y desafíos de la política social en México", Problemas del Desarrollo, 174 (44), pp. 9-34.

Palma, David y Arnulfo Sánchez (comps.) (2002), Plan de uso sustentable de los suelos de Tabasco, Vol. II, Tabasco, México, Colegio de PosgraduadosISPROTAB.

Peña, Sergio (2003), "Comercio transfronterizo y su impacto en la región de El Paso-Ciudad Juárez: Una propuesta de financiamiento de la planeación binacional”, Frontera Norte, 15(29), pp. 186-200.

Perroux, François (1955), "Note sur les notion de pole de croissance", Economie Apliquee, 7(1-2), pp. 307-320.

Piketty, Thomas (2014), El capital en el siglo XXI, México, Fondo de Cultura Económica.

Soria, Víctor (2000), "La inversión extranjera directa y el crecimiento económico de México", Ponencia presentada en el Seminario: La Escasez de Alimentos Básicos en México en el siglo xxi, México, IIEC-Unam.

Vázquez Barquero, Antonio (1990), "Dinámica regional y reestructuración productiva”, Revista Sociología del Trabajo, núm. 10, Siglo XXI, Madrid, España. 